\title{
Tracer microrheology of $\gamma$-dodecalactone induced gelation of aqueous starch dispersions
}

\author{
Cornelia Heinemann ${ }^{\mathrm{a}}$, Frédéric Cardinaux ${ }^{\mathrm{b}}$, Frank Scheffold ${ }^{\mathrm{b}}$, \\ Peter Schurtenberger $^{\mathrm{b}}$, Felix Escher ${ }^{\mathrm{a}}$, Béatrice Conde-Petit ${ }^{\mathrm{a}, *}$ \\ ${ }^{a}$ Laboratory of Food Chemistry and Technology, Institute of Food Science and Nutrition, Swiss Federal Institute of Technology (ETH) Zurich, \\ CH-8092 Zurich, Switzerland \\ ${ }^{\mathrm{b}}$ Department of Physics, University of Fribourg, CH-1700 Fribourg, Switzerland
}

Received 17 April 2003; revised 16 July 2003; accepted 8 September 2003

\begin{abstract}
We have studied the rheological properties of the natural biopolymer system starch in the presence of the gelation inducing flavor compound $\gamma$-dodecalactone. From optical microrheology, based on the multiple scattering of laser light (DWS), we deduce the frequency-dependent moduli $G^{\prime}(\omega)$ and $G^{\prime \prime}(\omega)$ of the gelling sample over the full frequency range. Our measurements demonstrate the inherent advantages of optical microrheology over classical mechanical experiments such as non-invasive sample access, extremely fast data acquisition time and an extended range of accessible frequencies. This enables us to study the critical power law scaling of $G^{\prime}(\omega)$ and $G^{\prime \prime}(\omega)$ of these weak physical gels in a single experimental run despite the fact that the gelation time is only of the order of a few minutes.
\end{abstract}

\section{Introduction}

Starch is widely used as thickening and gelling agent in food as well as in non-food applications. Heating of starch in presence of water induces swelling of starch granules and phase separation of the two starch polymers, amylose and amylopectin. This results in an increase of viscosity, which is also known as starch gelatinization. The rheological properties are largely determined by the volume fraction and the integrity of the starch granules. The disintegration of granular structure is favored by high temperatures, prolonged thermal treatment and mechanical energy input and is manifested by an exponential loss of viscosity (Conde-Petit, Nuessli, Handschin, \& Escher, 1998). Most starches contain around $25 \%$ of amylose, their behavior being crucial to the overall properties of the system. In neutral aqueous systems, amylose may be viewed as a highly disordered coil involving sequences of short-range, irregular and labile helical structures (Braga et al., 1985). The tendency of amylose to associate

*Corresponding author. Tel.: +41-1-632-37-31; fax: +41-1-632-11-23.

E-mail addresses: beatrice.conde@ilw.agrl.ethz.ch (B. Conde-Petit). through hydrogen bonding promotes the formation of interconnected networks, which finally results in macroscopic gelation. While the short-term changes of starch systems are governed by the aggregation of amylose, the reorganization of amylopectin is a much slower process that influences the long-term behavior of starch systems (Miles, Morris, Orford, \& Ring, 1985).

The properties of starch may be influenced at different levels of structure, ranging from nano to macroscale, by the addition of small molecules that are able to form helical starch inclusion complexes (Heinemann, Conde-Petit, Nuessli, \& Escher, 2001; Nuessli, Sigg, Conde-Petit, \& Escher, 1997). Suitable ligands are iodine, alcohols, fatty acids, emulsifiers, and different flavor compounds such as terpenes and lactones. The length of the helical domain depends on the size of the included ligand. For alcohols, a length of $10 \mathrm{~nm}$ has been reported (Jane \& Robyt, 1984). At the molecular level, complexing ligands induce the formation of $\mathrm{V}_{\mathrm{h}}$ amylose helices, the ligand being partially or completely included in the helical cavity in most cases. The strong tendency of the helical starch segments to aggregate results in the growth of supramolecular starch structures such as microscopic spherulites. 
At the macroscopic level bulk phase separation or gelation may be observed (Heinemann, Escher, \& Conde-Petit, 2003).

The sol-gel transition of starch, which occurs either as a consequence of the spontaneous aggregation of amylose or by the addition of complexing ligands, is of great practical importance. The sol-gel transition is characterized by the gel point, that is, the instant at which the connectivity of a network extends over the entire sample. The gel point is considered a material parameter, and its determination should not be influenced by the determination method (Flory, 1953). A widespread technique to follow gelation of colloidal polymer solutions is small amplitude oscillatory rheometry. The time at which $G^{\prime}$ and $G^{\prime \prime}$ curves cross each other has been proposed as a simple method for locating the gel point, but it depends on the frequency of the oscillation test (Tung \& Dynes, 1982). The preferred way to detect the gel point is the method of Winter and Chambon (1988), the criterion being that $G^{\prime \prime}(\omega) / G^{\prime}(\omega)=$ const. becomes independent of frequency over a wide range of frequencies at the gel point. From the slope of $G^{\prime}$ and $G^{\prime \prime}$ at the gel point, a relaxation exponent $n$ may be calculated. Its value ranges between 0 and 1 depending on the composition and on the molecular structure of the material (Scalan \& Winter, 1991). The validity of the Winter-Chambon method for accurately determining the gel point has been demonstrated in various studies including chemically and physically cross-linked gels (Rodd, Cooper-White, Dunstan, \& Boger, 2001; Ross-Murphy, 1995). However, the investigation of the gelation of weak physical gels by classical rheometry may be hampered by the fact that physical bonds are shear sensitive. Weak physical gels show a significant decrease of the critical strain, that is the limit of the linear viscoelastic region, close to the gel point (Rodd et al., 2001). Moreover the determination of the entire relaxation spectrum in a single run is most difficult and time consuming and in many cases even not feasible.

This study reports on the characterization of $\operatorname{starch} / \gamma$ dodecalactone systems with Diffusing Wave Spectroscopy (DWS)-based tracer microrheology. It complements a previous study on the influence of starch-lactone inclusion complexation on the formation of supramolecular structures in starch dispersion (Heinemann et al., 2003). It was shown that gelation of potato starch dispersions may be induced by starch/lactone complexation, the gelation time and the resulting gel strength being related to the length of the linear chain of the lactone molecule.

The aim of the present study was to follow the viscoelastic properties of potato starch dispersions upon complexation with $\gamma$-dodecalactone as a function of time with emphasis on the sol-gel transition. The underlying principle of optical tracer microrheology is to follow the average Brownian fluctuation (thermal motion) of particles artificially inserted into medium under study (Mason, Ganesan, van Zanten, Wirtz, \& Kuo, 1997a; Mason, Gang, \& Weitz, 1997b; Mason \& Weitz, 1995).
From a generalized Stokes-Einstein relation (Levine \& Lubensky, 2000, 2001), the measured particle mean-square displacement (MSD) can be linked to the storage, $G^{\prime}$, and loss, $G^{\prime \prime}$, modulus. In fact, the main advantage of DWS-based microrheology is that a broad frequency range in $G^{\prime}(\omega)$ and $G^{\prime \prime}(\omega)$ is accessible, which cannot be covered with classical rheometer. In the latter, this is demonstrated by a comparison of the microrheology results with the macroscopic viscoelastic properties obtained from small amplitude oscillatory shear rheology over the frequency range accessible to our rheometer $(\sim \omega<25 \mathrm{~Hz})$.

The experiments were carried out with potato starch dispersion at low concentration $(1.9 \mathrm{~g} / 100 \mathrm{~g}$ dispersions $)$ that can be considered as an aqueous model food system, where starch is used as thickener. Potato starch was selected since it is free of internal lipids and the occurrence of spontaneous crystallization is retarded at low concentrations.

\section{Materials and methods}

\subsection{Material}

Native potato starch was obtained from Blattmann \& Co., $\mathrm{CH}-\mathrm{Wädenswil.} \mathrm{The} \mathrm{ligand} \gamma$-dodecalactone was supplied by Fluka, CH-Buchs.

\subsection{Sample preparation}

The starch dispersions were prepared by heating of native potato starch in water. The temperature, time and mixing conditions were selected so that amylose was completely solubilized as assessed by iodine titration (Conde-Petit et al., 1998; Heinemann et al., 2003). For this purpose, aqueous suspensions of native potato starch at a concentration of $2 \mathrm{~g}$ dry starch/100 g suspension were filled in cans and heated in a retort (Berlin Chapman, WI, USA) at 1 bar overpressure $\left(121^{\circ} \mathrm{C}\right)$ for $30 \mathrm{~min}$. After $30 \mathrm{~min}$, the starch dispersion in the cans had reached a temperature of $112^{\circ} \mathrm{C}$ (Conde-Petit et al., 1998). Thereafter, the cans were cooled to $30^{\circ} \mathrm{C}$ within $10 \pm 2 \mathrm{~min}$ and stored for 1 week at room temperature. The process led to an almost complete loss of granular structure as revealed by optical microscopy. During aging of the starch dispersion in the cans, no aggregation or recrystallization of starch occurred as probed by amperometric iodine titration, rheological measurements and X-ray diffraction measurements (Heinemann et al., 2003)

A sulfate latex solution of polystyrene spheres $(\varnothing$ 720 nm) (Interfacial Dynamics Corporation, USA-Portland) at a concentration of $8.2 \mathrm{~g} / 100 \mathrm{~g}$ solution was added to the starch dispersion so that the final concentration was $0.41 \mathrm{~g}$ latex/100 $\mathrm{g}$ dispersion ensuring strong multiple scattering of visible light. The polystyrene spheres act as tracer particles to probe the local viscoelastic properties by Brownian 
motion. The size of the particles is chosen such that the typical relevant length scale $\xi$ (several nanometers) of the biopolymer solutions is much smaller than the particle diameter $\varnothing 720 \mathrm{~nm}$.

The final starch concentration in the system was $1.9 \mathrm{~g} / 100 \mathrm{~g}$ dispersion. $\gamma$-Dodecalactone was added to the starch dispersions at saturation concentration of the amylose that corresponds to $50 \mathrm{mmol} / \mathrm{mol}$ anhydroglucose. The molecular starch concentration is based on the molecular weight of anhydroglucose $\left(\mathrm{C}_{6} \mathrm{H}_{10} \mathrm{O}_{5}\right)$ instead of an average molecular weight of starch. The flavor substances were weighed into jars and starch dispersion at room temperature was added. The jars were closed and the mixture was shaken for about $20 \mathrm{~s}$. The selected ligand concentration allowed full complexation of amylose as probed by iodometric titration (Heinemann et al., 2003). The starch- $\gamma$-dodecalactone system and a polystyrene sphere are schematically presented in Fig. 1.

\subsection{Small amplitude oscillatory shear measurements}

Oscillatory shear measurements were using a controlled stress rheometer (Carri-Med CSL 100, GB-Surrey) with a cone and plate geometry $\left(\varnothing 6 \mathrm{~cm}\right.$, angle $1^{\circ} 59 \mathrm{~min}$, gap $55 \mu \mathrm{m})$. To follow the sol-gel transition, the starch dispersion was transferred to the rheometer immediately after $\gamma$-dodecalactone addition. The storage $\left(G^{\prime}\right)$ and loss modulus $\left(G^{\prime \prime}\right)$ were followed during $1 \mathrm{~h}$ at a temperature of $25 \pm 0.1^{\circ} \mathrm{C}$, at a frequency of $1 \mathrm{~Hz}$ and a stress of $0.02 \mathrm{~Pa}$ which was within the linear viscoelastic range. In one case, the frequency dependence of $G^{\prime}$ and $G^{\prime \prime}$ of potato starch dispersions was determined between 1 and $25 \mathrm{~Hz}$.

To investigate the influence of tracer particles on the rheological behavior of the starch system, potato starch dispersions with and without polystyrene spheres were examined with small amplitude oscillatory shear measurements. The time dependence of the viscoelastic moduli of potato starch dispersion without addition and with $\gamma$-dodecalactone, with and without polystyrene spheres during an aging time of $1 \mathrm{~h}$ is presented in Fig. 2.

Potato starch dispersions without addition of $\gamma$-dodecalactone (with and without polystyrene spheres) were liquidlike and presented a loss modulus $G^{\prime \prime}$ that was higher than the storage modulus $G^{\prime}$. Both systems showed the same viscoelastic properties. The viscoelastic moduli did not change during aging time indicating that the amylose did not aggregate during the experiment. The time dependence of the viscoelastic moduli are in good agreement with those of fresh potato starch dispersion as shown by Heinemann et al. (2003). The addition of $\gamma$-dodecalactone to the starch dispersion without polystyrene spheres led to an increase of the storage modulus $G^{\prime}$, the transition of the storage modulus $G^{\prime}$ and loss modulus $G^{\prime \prime} \sim 6$ min after sample preparation marking the sol-gel transition. It seems that the addition of polystyrene spheres to starch dispersion with $\gamma$-dodecalactone accelerates somewhat the solidification of the material. It is, however, difficult to quantify this effect since the time to prepare a sample and the gel time are of the same order of a few minutes. In any case, we do not expect the tracer particles to influence neither the process of

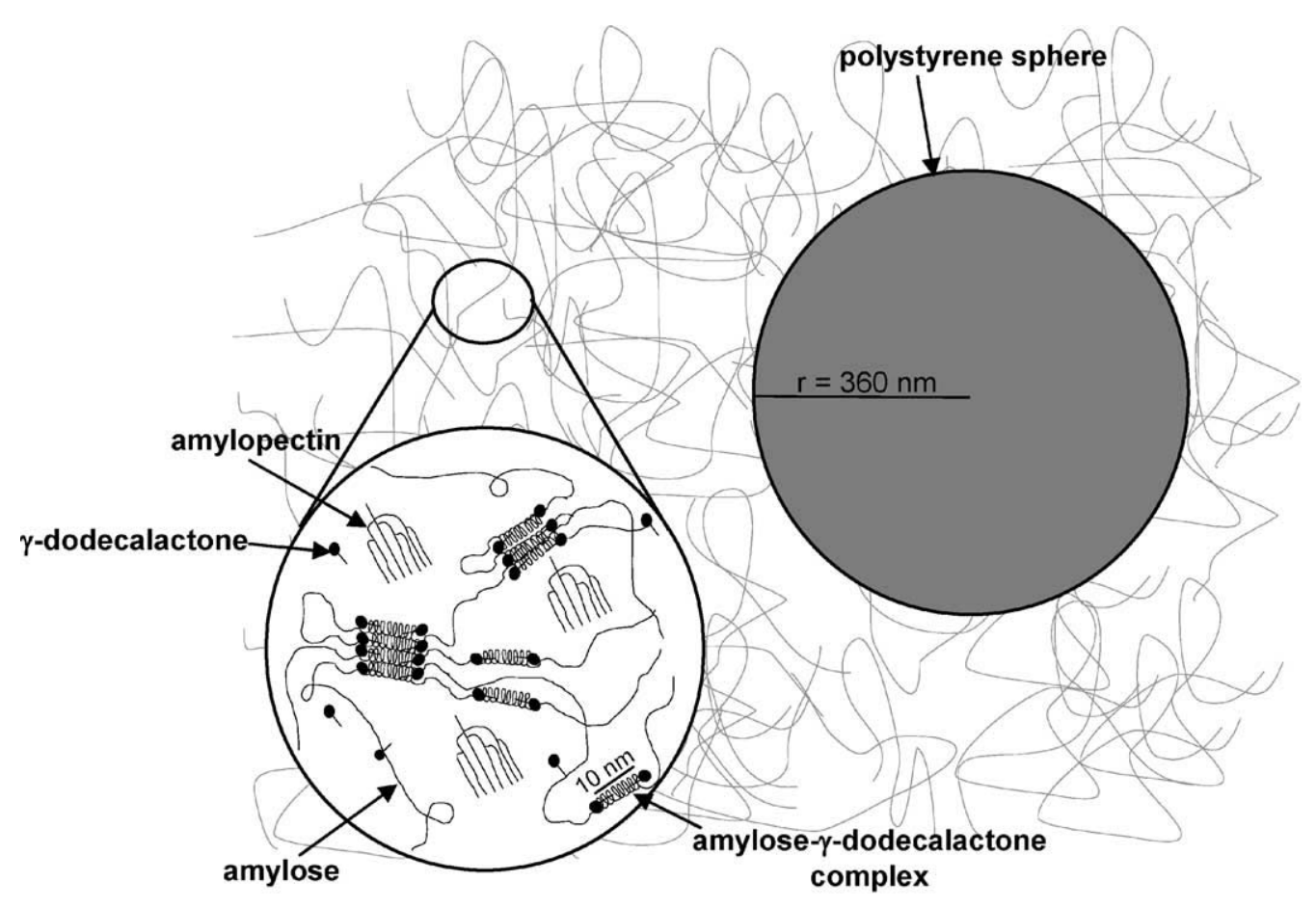

Fig. 1. Schematic drawing of the polystyrene sphere and the starch- $\gamma$-dodecalactone system. 

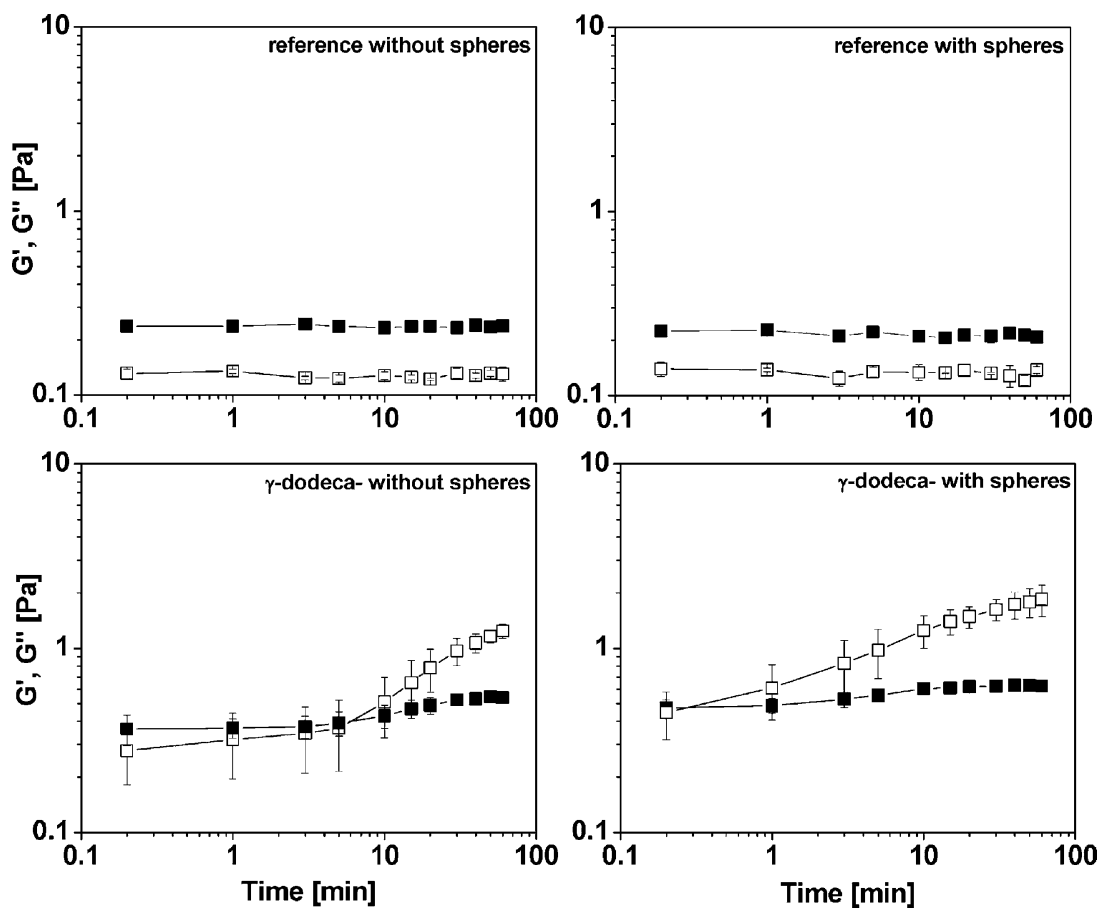

Fig. 2. Time dependence of the storage modulus $G^{\prime}$ (open square) and loss modulus $G^{\prime \prime}$ (closed square) of potato starch dispersion without addition and with $\gamma$-dodecalactone, with and without polystyrene spheres measured with oscillatory shear measurements.

the gelation itself nor the strength of the gel at sufficiently long times due to their low weight fraction of only $0.41 \%$.

\subsection{Diffusing wave spectroscopy}

DWS is an extension of dynamic light scattering (DLS) to soft materials exhibiting strong multiple scattering (Maret \& Wolf, 1987). It has been shown that DWS allows monitoring the displacement of micron-sized colloidal particles with nanometer precision of better. In recent years, significant progress has been made in development and further understanding of the DWS approach. Over the last decade, it has been applied for the study of fluid and solid media such as colloidal suspensions and gels, biocolloids (yoghurt and cheese) as well as ceramic slurries and green bodies (Dagleish \& Horne, 1991; Grotenhuis, Paques, \& van Aken, 2000; Romer, Scheffold, \& Schurtenberger, 2000; Scheffold \& Schurtenberger, 2003; Schurtenberger, Stradner, Romer, Urban, \& Scheffold, 2001; Wyss, Romer, Scheffold, Schurtenberger, \& Gauckler, 2001).

In a DWS experiment, coherent laser light impinges on one side of a turbid sample and the intensity fluctuations of the light propagated through the sample are then analyzed. A diffusion model is used to describe the propagation of photons across the sample. The diffusion approximation allows to determine the distribution of scattering paths and to calculate the temporal autocorrelation of the intensity fluctuations. DWS provides quantitative information on the average MSD of the scattering particles from the measured intensity autocorrelation function over a very broad range of time scales.

Analogous to DLS, it is possible to express the measured intensity autocorrelation function $g_{2}(\tau)-1=\langle I(t) I(t+\tau)\rangle /\langle I\rangle^{2}-1$ in terms of the MSD of the scattering particle

$g_{2}(\tau)-1=\left[\int_{0}^{\infty} \mathrm{d} s P(s) \exp \left(-\left(s / l^{*}\right) k^{2}\left\langle\Delta r^{2}(\tau)\right\rangle\right)\right]^{2}$

with $k=2 \pi n / \lambda$ being the wave number of light in a medium with refractive index $n . P(s)$ is the distribution of photon trajectories of length $s$ in the sample and it can be calculated within the diffusion model taking into account the experimental geometry. The transport mean free path $l^{*}$ characterizes the typical step length of the photon random walk, given by the individual particle scattering properties and particle concentration. $l^{*}$ can be determined independently and enters the analysis as a constant parameter. From Eq. (1), it is possible to numerically calculate the particle $\operatorname{MSD}\left\langle\Delta r^{2}(\tau)\right\rangle$ from the measured autocorrelation function $g_{2}(\tau)$. From the particle MSD, the frequency-dependent storage and loss moduli $G^{\prime}$ and $G^{\prime \prime}$ can be directly determined. The details of this procedure can be found in literature (Mason et al., 1997a; Mason et al., 1997b).

We used a combination of transmission and backscattering measurements as described in Cardinaux, Cipelletti, Scheffold, and Schurtenberger (2002) to obtain simultaneous access to fast dynamics (in transmission) and slow dynamics (in backscattering). Fig. 3 presents a schematic view of the DWS set-up. A laser beam at 


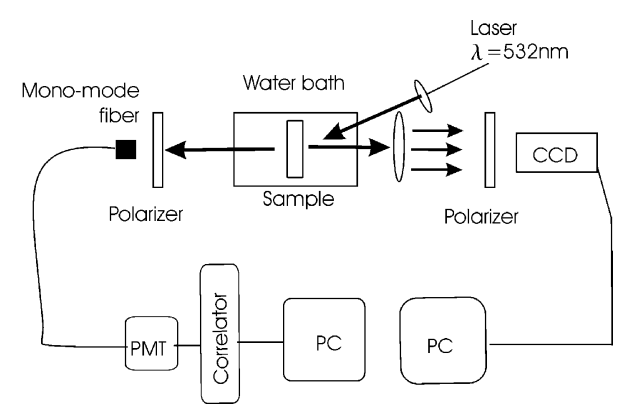

Fig. 3. DWS set-up: an intense laser beam (Verdi from Coherent) is scattered from a turbid sample contained in a temperature controlled water bath. The scattered light is detected in transmission or backscattering with a single mode fiber or a CCD camera and subsequently analyzed digitally (correlator and PC).

a wave length $\lambda$ of $532 \mathrm{~nm}$ is scattered from a turbid sample contained in a temperature controlled water bath at $25^{\circ} \mathrm{C}$. The scattered light is detected with a mono mode fiber in transmission and a CCD camera in backscattering and subsequently analyzed digitally (correlator and PC). The thickness of the cell was $1 \mathrm{~cm}$ to ensure multiple scattering of light for the chosen particle density $(0.41 \mathrm{~g}$ latex/100 $\mathrm{g}$ dispersion). To obtain the frequency-dependent viscoelastic moduli, we applied the procedure described in Mason et al. (1997a), which leaves the experimentally obtained $G^{\prime}(\omega)$ and $G^{\prime \prime}(\omega)$ free of any adjustable parameter or fits contrary to the method initially suggested by Mason and Weitz (1995) and also applied by Cardinaux et al. (2002) based on a polynomial fit of $\left\langle\Delta r^{2}(\tau)\right\rangle$.

\section{Results and discussion}

The temporal dependence of the MSD $\left(=\left\langle\Delta r^{2}\right\rangle\right)$ of scattering polystyrene spheres embedded in potato starch dispersion complexed with $\gamma$-dodecalactone 5, 15, $70 \mathrm{~min}$ and $23 \mathrm{~h}$ after complexation is presented in Fig. 4.

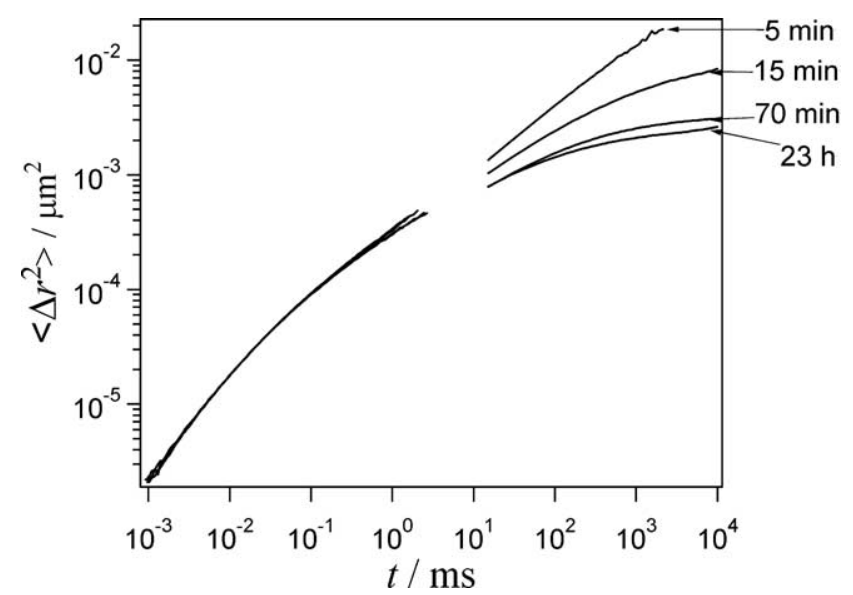

Fig. 4. Mean-square displacement of spherical polystyrene particles embedded in potato starch dispersion complexed with $\gamma$-dodecalactone after 5, 15, $70 \mathrm{~min}$ and $23 \mathrm{~h}$.
The most important changes in the MSD occurred during the first $70 \mathrm{~min}$ after complexation. Before adding the ligands, classical Brownian diffusion $\left(\left\langle\Delta r^{2}\right\rangle=6 D t\right.$, with a particle diffusion constant $D$ ) of the polystyrene spheres over the accessible time range was observed as manifested by the linear dependence of the MSD on time (data not shown). Already $5 \mathrm{~min}$ after complexation of the potato starch dispersion with $\gamma$-dodecalactone, the mobility of the embedded particles is visibly restricted at long times. The subsequent occurrence of a plateau in the MSD reflects the formation of an interconnected network (gel) induced by amylose- $\gamma$-dodecalactone complexation. As a consequence, the trapped polystyrene spheres only executed spatially limited motions about their fixed averaged positions. Only a small change of the maximum MSD (plateau value) was observed $70 \mathrm{~min}$ to $23 \mathrm{~h}$ after complexation of potato starch dispersion with $\gamma$-dodecalactone.

The frequency dependence of the storage and the loss moduli of potato starch dispersion complexed with $\gamma$-dodecalactone 5, 15, $70 \mathrm{~min}$ and $23 \mathrm{~h}$ after complexation is shown in Fig. 5. Fig. 5 additionally presents the frequency dependence of the mature gel $70 \mathrm{~min}$ after complexation measured with small amplitude oscillatory rheometry. Initially the characteristic behavior of a liquid sample is observed. $5 \mathrm{~min}$ after complexation, the loss and storage moduli obey the same power law over a broad frequency range with an exponent $n=0.5$. Following the criterion of Winter and Chambon (1988), this time corresponds to the gel point $\left(t_{\mathrm{C}}\right)$, marking the transition from a liquid- to
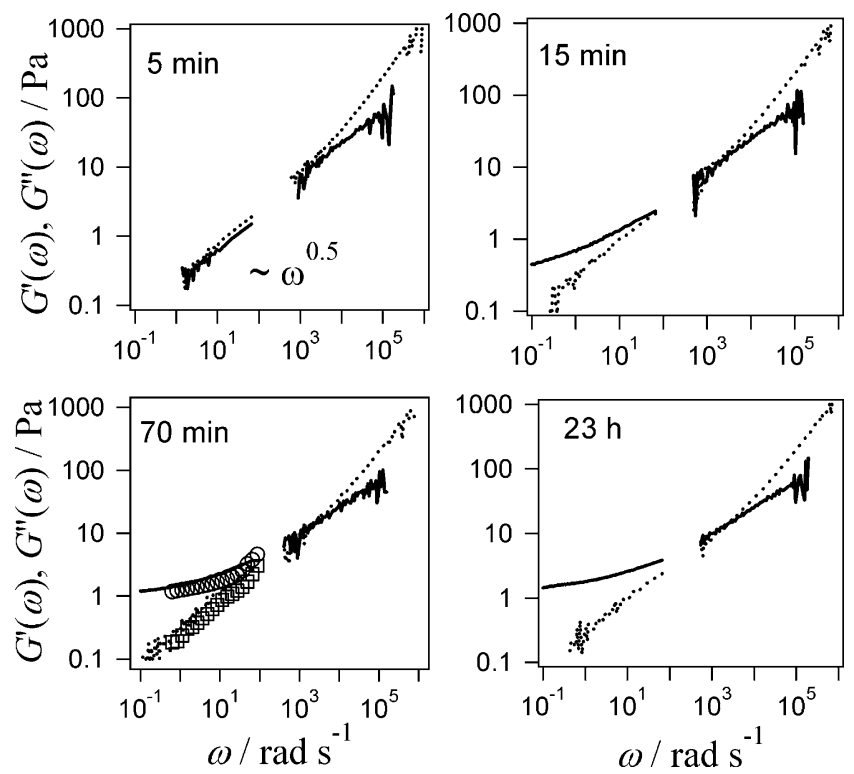

Fig. 5. Frequency dependence of the storage (straight line) and the loss moduli (dotted line) of potato starch dispersion complexed with $\gamma$-dodecalactone after an aging time of 5, 15, $70 \mathrm{~min}$ and $23 \mathrm{~h}$ measured with DWS and compared to the storage modulus $G^{\prime}$ (open circle) and loss modulus $G^{\prime \prime}$ (open square) of classical rheological measurements after an aging time of $70 \mathrm{~min}$. 
a solid-like behavior. For times smaller than $t_{\mathrm{C}}$, that is before gelation, the curves slope downwards at low frequencies, which is indicative of fluid-like behavior (data not shown). At times after the gel point, $G^{\prime}$ flattened at low frequency-a characteristic of solid-like behavior. After $23 \mathrm{~h}$, the starch dispersion was clearly elastic, with the storage modulus showing a clear low frequency plateau. As the gel matured, the plateau height increased and the cross-over point (of $G^{\prime}$ and $G^{\prime \prime}$ ) moved to higher frequency and higher moduli. For high frequency, the loss modulus asymptotically approached a power law frequency dependence. Good agreement was found between DWS-based microrheology and small amplitude oscillatory shear measurements in the low frequency range where both methods overlap.

From our measurements, it is clearly seen that the combination of DWS measurements in the transmission and backscattering geometry provide access to small and long time scales, respectively. Note that the gap in the $G^{\prime}$ and $G^{\prime \prime}$ at $\omega \sim 500 \mathrm{~Hz}$ (see Fig. 5) is due to the fact that the accessible time scales for the transmission and backscattering mode do not overlap in our experiments. This is due to the limited time resolution of the CCD camera and the short experimental runs of only about $1 \mathrm{~min}$ (limiting the correlation times accessible for single photon counting to a few ms) as seen also in Fig. 4. On the other hand, the short data acquisition time for DWS measurements of a minute or less are highly advantageous since time-dependent changes of the mechanical properties on a minute scale can be resolved while still covering a frequency range of up to six decades $\left(\omega \sim 1-10^{6} \mathrm{~Hz}\right)$. Furthermore, the technique always works in thermal equilibrium hence no structural changes or distortions of the systems arise due to the application of external shear forces.

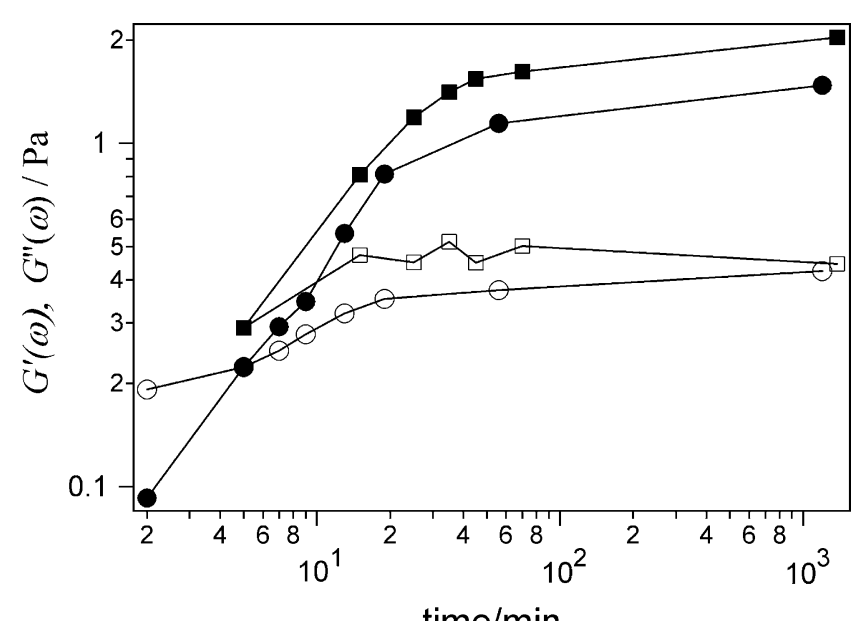

time/min

Fig. 6. Reproducibility of optical microrheology measurements: Time dependence of the low frequency storage modulus $G^{\prime}$ (filled symbol) and loss modulus $G^{\prime \prime}$ (open symbol) taken at $2 \mathrm{rad} / \mathrm{s}$ from two different experimental runs.
Finally, the reproducibility of the viscoelastic moduli $G^{\prime}$ and $G^{\prime \prime}$ as calculated from DWS measurements of potato starch dispersion complexed with $\gamma$-dodecalactone is presented in Fig. 6. The experiment was started after mixing of the starch dispersion with the ligand which was after 2 and $\sim 4$ min, respectively. The measurements showed good reproducibility and are in good agreement with results obtained with classical rheological measurements by Heinemann et al. (2003).

\section{Conclusions and outlook}

DWS-based tracer microrheology is suitable for the following rheological changes of starch dispersion as induced by helical inclusion complexation. The experimental set-up allows to simultaneously capture the fast and slow dynamics of the system and thus, to follow the viscoelastic moduli covering a frequency range of six decades. Using DWS as an optical probe of local tracer particle motion provides non-invasive access to the linear viscoelastic properties of the gelling sample at any time during the gelation process. We have demonstrated that an accurate determination of the gel point of starch dispersions is possible based on the power law criterion of Winter and Chambon (1988). Good agreement was found between DWS-based microrheology and small amplitude oscillatory shear measurements in the low frequency range. The detailed information about $G^{\prime}(\omega)$ and $G^{\prime \prime}(\omega)$ now available provides a new basis to describe quantitatively changes in starch polymer conformation upon gelation. Thereby, a better understanding of the molecular and supramolecular changes underlying the sol-gel transition can be obtained.

\section{Acknowledgement}

Financial support from the Swi National Science Foundation is gratefully acknowledged.

\section{References}

Braga, D., Ferracini, E., Ferrero, A., Ripamoti, A., Brant, D. A., Buliga, G. S., \& Cesaro, A. (1985). Amylose conformation in aqueous solution-a small-angle X-ray scattering study. International Journal of Biological Macromolecules, 7, 161-166.

Cardinaux, F., Cipelletti, L., Scheffold, F., \& Schurtenberger, P. (2002). Microrheology of giant-micelle solutions. Europhysics Letters, 57, $738-744$.

Conde-Petit, B., Nuessli, J., Handschin, S., \& Escher, F. (1998). Comparative characterisation of aqueous starch dispersions by light microscopy, rheometry and iodine binding behavior. Starch/Stärke, 50, $184-192$.

Dagleish, D. G., \& Horne, D. S. (1991). Studies of gelation of acidified milk using diffusing wave spectroscopy. Milchwisenschaft-Milk Science International, 46(7), 417. 
Flory, P. J. (1953). Principles of polymer chemistry. Ithaca: Cornell University Press.

Grotenhuis, E., Paques, M., \& van Aken, G. A. (2000). The application of diffusing-wave spectroscopy to monitor phase behavior of emulsionpolysaccharide systems. Journal of Colloid and Interface Science, 227, 495-504.

Heinemann, C., Conde-Petit, B., Nuessli, J., \& Escher, F. (2001). Evidence of starch inclusion complexation with lactones. Journal of Agricultural and Food Chemistry, 49, 1370-1376.

Heinemann, C., Escher, F., \& Conde-Petit, B. (2003). Structural features of starch-lactone inclusion complexes in aqueous potato starch dispersions: The role of amylose and amylopectin. Carbohydrate Polymers, $51,159-168$.

Jane, J.-L., \& Robyt, F. (1984). Structure studies of amylose-V complexes and retrograded amylose by action of alpha amylases, and a new method for preparing amylodextrins. Carbohydrate Research, 132, $105-118$.

Levine, A. J., \& Lubensky, T. C. (2000). One- and two-particle microrheology. Physical Review Letters, 85, 1774-1777.

Levine, A. J., \& Lubensky, T. C. (2001). Response function of a sphere in a viscoelastic two-fluid medium. Physical Review E, 63(041510), 1-4.

Maret, G., \& Wolf, P. E. (1987). Multiple light-scattering from disordered media - the effect of Brownian motion of scatteres. Zeitschrift für Physik B-Condensed Matter, 65, 409-413.

Mason, T. G., Ganesan, K., van Zanten, J. H., Wirtz, D., \& Kuo, S. C. (1997a). Particle tracking microrheology of complex fluids. Physical Review Letters, 79, 3282-3285.

Mason, T. G., Gang, H., \& Weitz, D. A. (1997b). Diffusing-wavespectroscopy measurements of complex fluids. Journal of the Optical Society of America A-Optics Image Science and Vision, 14, 139-149.

Mason, T. G., \& Weitz, D. A. (1995). Optical measurements of frequencydependent linear viscoelastic moduli of complexing fluids. Physical Review Letters, 74, 1250-1253.
Miles, M. J., Morris, V. J., Orford, P. D., \& Ring, S. G. (1985). The role of amylose and amylopectin in the gelation and retrogradation of starch. Carbohydrate Research, 135, 271-281.

Nuessli, J., Sigg, B., Conde-Petit, B., \& Escher, F. (1997). Characterization of amylose-flavor complexes by DSC and X-ray diffraction. Food Hydrocolloids, 11, 27-34.

Rodd, A. B., Cooper-White, J., Dunstan, D. E., \& Boger, D. V. (2001). Gel point studies for chemically modified biopolymer networks using small amplitude oscillatory rheometry. Polymer, 42, 185-198.

Romer, S., Scheffold, F., \& Schurtenberger, P. (2000). Sol-gel transition of concentrated colloidal suspensions. Physical Review Letters, 85, 4980-4983.

Ross-Murphy, S. B. (1995). Rheological characterization of gels. Journal of Texture Studies, 26, 391-400.

Scalan, J. C., \& Winter, H. H. (1991). Composition dependence of the viscoelasticity of end-linked poly(dimethylsiloxane) at the gel point. Macromolecules, 24, 47-54.

Scheffold, F., \& Schurtenberger, P. (2003). Light scattering probes of viscoelastic fluids and solids. Soft Materials, 1, 139-165.

Schurtenberger, P., Stradner, A., Romer, S., Urban, C., \& Scheffold, F. (2001). Aggregation and gel formation in biopolymer solutions. Chimia, 55, 155-159.

Tung, C. Y. M., \& Dynes, P. J. (1982). Relationship between viscoelastic properties and gelation in thermosetting systems. Journal of Applied Polymer Science, 27, 569-574.

Winter, H. H., \& Chambon, F. (1988). Analysis of linear viscoelasticity of a crosslinking polymer at the gel point. Journal of Rheology, 30, 367-382.

Wyss, H., Romer, S., Scheffold, F., Schurtenberger, P., \& Gauckler, L. J. (2001). Diffusing wave spectroscopy of concentrated alumina suspensions during gelation. Journal of Colloidal and Interface Science, 241, 89-97. 